\title{
Tasting edge effects
}

\author{
Lydéric Bocquet* \\ Laboratoire de Physique de Physique de la Matière Condensée et Nanostructures, \\ Université Lyon 1, UMR CNRS 5586, 43, Bd du 11 Novembre 1918, 69622 Villeurbanne France
}

\begin{abstract}
We show that the baking of potato wedges constitutes a crunchy example of edge effects, which are usually demonstrated in electrostatics. A simple model of the diffusive transport of water vapor around the potato wedges shows that the water vapor flux diverges at the sharp edges in analogy with its electrostatic counterpart. This increased evaporation at the edges leads to the crispy taste of these parts of the potatoes.
\end{abstract}

\section{INTRODUCTION}

Edge effects are usually introduced in electrostatic courses and provide an interesting and nontrivial example of electrostatic effects. This phenomenon corresponds to the divergence of the electric field and charge accumulation at the edges or corners of a conductor at a fixed potential. This singular behavior has various consequences and applications such as lightning rods and the field emission effect.

The mathematical description of edge effect involves the solution of the Laplace equation for the electric potential with fixed potential boundary conditions on the conductor. For example, for a corner with an opening angle $\alpha$ in a two-dimensional geometry, the electric field $E$ and surface charge on the conductor behaves as $E \propto \rho^{\gamma}$ with $\gamma=(\alpha-\pi) /(2 \pi-\alpha)$ and $\rho$ the distance to the tip. 1 The electric field thus diverges at the corners when $\alpha<\pi$.

Alternative examples of edge effects can be found in other domains of physics. The minimal ingredients are a geometry with sharp edges; a Laplace-like equation for the physical quantity of interest (for example, the electric potential); and a boundary condition on the given geometry that imposes a fixed value of this quantity at its surface.

Evaporation of water vapor is one such example, as we will discuss in the following. Edge effects arise in the context of molecular diffusion of water vapor. A diverging water vapor flux at the edges is predicted. Such effects have been shown to be responsible for the formation of ring stains formed by drying coffee drops. ${ }^{2}$

We consider another example of an edge effect induced by evaporation: the drying of potato wedges baked in an oven. The geometry of the potatoes is fixed by the cook: we shall focus here on potatoes cut with sharp edges, as for potato wedges. We show in Fig. 1 an example of (home-made) potato wedges (after 20 minutes in the oven at $200^{\circ} \mathrm{C}$ ). As can be seen the edges are much darker, and exhibit very strong drying.

We will demonstrate that this drying is due to a diverging flux of water vapor at the extremities of the potato wedge in analogy with the edge effect in electrostatics. This divergence induces a strong dehydration of the potatoes in its wedges and corners.

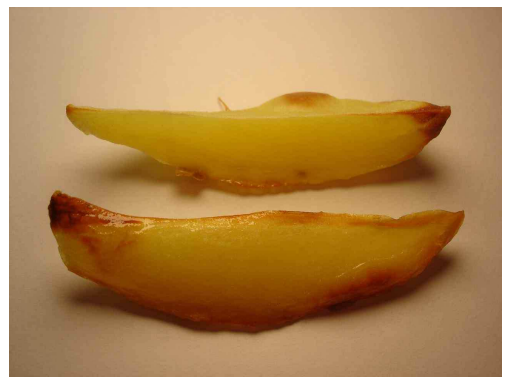

FIG. 1: Two (home-made) baked potato wedges. The edges are seen to be darker, showing dehydration in these regions. The effect is stronger at the extremities of the wedge.

\section{WATER VAPOR DIFFUSION AROUND POTATOES}

Like most foods, potatoes contain a large amount of water. While increasing the temperature in the oven, the liquid-vapor thermodynamic equilibrium of water is displaced toward the vapor phase, which leads to the evaporation of the liquid water inside the potatoes to the surrounding air.

Let us consider the distribution of water vapor in the air around potatoes. Its concentration $c_{\mathrm{w}}$ obeys a diffusion equation:

$$
\frac{\partial c_{\mathrm{w}}}{\partial t}=D_{v} \nabla^{2} c_{\mathrm{w}}
$$

with $D_{v}$ the diffusion coefficient of the water vapor in air. We make the further assumption that the oven is in a quasi-stationary state, which implies that

$$
\nabla^{2} c_{\mathrm{w}}=0
$$

On the potato wedge surface, the value of the vapor concentration is fixed by the liquid-vapor thermodynamic equilibrium, and equals the saturation value $c_{\text {sat }}$ calculated at the temperature of the oven. Far from the potato, we expect that the air in the oven is not saturated and the concentration of vapor reaches a fixed value $c_{\infty}$ lower than saturation. Because we are interested in the diffusion of water in the vicinity of the potato's surface, this far field boundary condition will not be required to 
characterize edge effects. The rate of evaporation of water at the potato's surface is given by the flux of water vapor, $J_{D}=-D_{v} \nabla c_{\mathrm{w}}$.

The equation for the vapor concentration with the specified boundary conditions is identical to that of the electrostatic potential around a conductor at fixed potential. The solution of Eq. (2) depends only on the geometry of the conductor/potato's surface $\stackrel{1.2}{ }$ The vapor flux is analogous to the electric field and is therefore expected to diverge at the edges. Both the edges and extremities of a potato will be considered: these will be modeled respectively in terms of two- and three-dimensional geometries. We now recall the basic steps in deriving the solution of Eq. (2) in the context of our problem. A detailed description can be found in Ref. 1 .

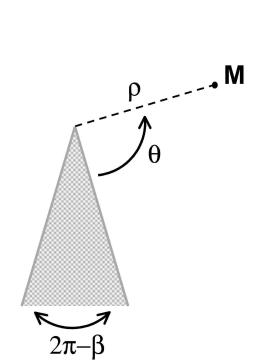

(a)

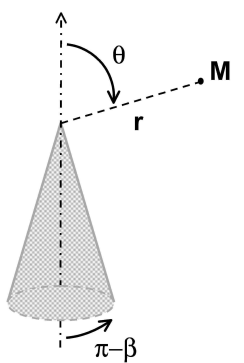

(b)
FIG. 2: Geometry of the system: (a) two-dimensional wedge; (b) three-dimensional cone.

\section{DRYING OF WEDGES: TWO-DIMENSIONAL GEOMETRY}

First consider a two-dimensional geometry as in Fig. 2(a). This geometry corresponds to evaporation at the border of a potato's wedge. The Laplace equation for the vapor concentration is

$$
\frac{1}{\rho} \frac{\partial}{\partial \rho}\left(\rho \frac{\partial c_{\mathrm{w}}}{\partial \rho}\right)+\frac{1}{\rho^{2}} \frac{\partial^{2} c_{\mathrm{w}}}{\partial \theta^{2}}=0 .
$$

We look for a solution using separation of variables, $c_{\mathrm{w}}=$ $f(\rho) g(\theta)$, and find the equations for $f$ and $g$ :

$$
\begin{aligned}
\frac{\rho}{f} \frac{\partial}{\partial \rho}\left(\rho \frac{\partial f}{\partial \rho}\right) & =K^{2} \\
\frac{1}{g} \frac{\partial^{2} g}{\partial \theta^{2}} & =-K^{2}
\end{aligned}
$$

with solutions $f(\rho)=a_{K} \rho^{K}+b_{K} \rho^{-K}, g(\theta)=$ $A_{K} \sin (K \theta)+B_{K} \cos (K \theta)$ for $K \neq 0$ and $f(\rho)=a_{0}+$ $b_{0} \log \rho, g(\theta)=A_{0}+B_{0} \theta$ for $K=0$.

The constant $K$ is fixed by the boundary conditions on the wedge's surface. To avoid a divergence of the concentration at the surface, we need to impose $b_{0}=$ $b_{K}=0$. Imposing $c_{\mathrm{w}}=c_{\mathrm{sat}}$ for $\theta=0$ and $\theta=\beta(\rho>0)$ yields $a_{0}=c_{\text {sat }}, B_{0}=B_{K}=0$. This condition also requires that $K$ satisfy $\sin (K \beta)=0$, so that $K=n \pi / \beta$, with $n=1,2, \ldots$. We put these results together and write the vapor concentration as

$$
c_{\mathrm{w}}(\rho, \theta)=c_{\mathrm{sat}}+\sum_{n=1}^{\infty} a_{n} \rho^{n \pi / \beta} \sin \left(\frac{n \pi \theta}{\beta}\right) .
$$

The coefficients $a_{n}$ are determined from the concentration far from the corner, and it is not necessary to determine their value for the discussion of the edge effects..

The water flux at the surface of the potato can now be determined using $J_{D}=-D_{v} \nabla c_{\mathrm{w}}$. Only the component of the flux perpendicular to the surface is non-vanishing. For $\theta=0$, we obtain:

$$
J_{D}=-D_{v} \sum_{n=1}^{\infty} a_{n} \frac{n \pi}{\beta} \rho^{n \pi / \beta-1} .
$$

The first term in the sum is dominant close to the wedge, and the water flux behaves as

$$
J_{D} \approx-D_{v} \frac{a_{1} \pi}{\beta} \rho^{\pi / \beta-1} .
$$

As expected, this flux diverges at the edge for $\beta>\pi$, that is, for a sharp wedge. The exponent of the divergence $\pi / \beta-1$ takes its maximum value, $-1 / 2$, in the very sharp limit, $\beta \rightarrow 2 \pi$. Note that the same exponent $(1 / 2)$ is found for the divergence of the surface charge close to the edge of a thin disk at fixed potential. 1

\section{DRYING OF EDGES: THREE-DIMENSIONAL GEOMETRY}

Our derivation can be generalized to the conical shape shown in Fig. 2(b) $\stackrel{4}{=}$ If we use spherical coordinates and assume azimutal symetry, the Laplace equation becomes

$$
\frac{1}{r} \frac{\partial^{2} r c_{\mathrm{w}}}{\partial r^{2}}+\frac{1}{r^{2} \sin \theta} \frac{\partial}{\partial \theta}\left(\sin \theta \frac{\partial c_{\mathrm{w}}}{\partial \theta}\right)=0 .
$$

We look for a solution of the form $c_{\mathrm{w}}=\frac{f(r)}{r} g(\theta)$ and obtain the following equations for $f(r)$ and $g(\theta)$ :

$$
\begin{aligned}
\frac{\partial^{2}}{\partial r^{2}} f-K^{\prime} \frac{f}{r^{2}} & =0 \\
\frac{1}{\sin \theta} \frac{\partial}{\partial \theta}\left(\sin \theta \frac{\partial g}{\partial \theta}\right)+K^{\prime} g & =0 .
\end{aligned}
$$

It is convenient to rewrite the unknown constant $K^{\prime}$ as $\ell(\ell+1)$. The solution for $f$ can be written as $f(r)=$ $a r^{\ell+1}+b r^{-\ell}$; the solution for $g(\theta)$ is the Legendre function of the first kind of order $\ell, P_{\ell}(\cos \theta) \stackrel{3}{\underline{3}}$ The regularity of the solution at the origin imposes $b=0$. The boundary condition at the surface of the potato, $c_{\mathrm{w}}=c_{\mathrm{sat}}$, leads to the following condition for the index $\ell$ :

$$
P_{\ell}(\cos \beta)=0
$$


There is an infinite number of solutions for Eq. (10), which we denote as $\ell_{n}$, with $n=1,2, \ldots$ Following the same steps as for the two-dimensional case, we obtain the general solution as a linear combination of solutions:

$$
c_{\mathrm{w}}(r, \theta)=c_{\mathrm{sat}}+\sum_{n=1}^{\infty} a_{n} r^{\ell_{n}} P_{\ell_{n}}(\cos \theta) .
$$

The water flux on the surface thus takes the form:

$$
J_{D}=D_{v} \sum_{n=1}^{\infty} a_{n} r^{\ell_{n}-1} \sin \beta P_{\ell_{n}}^{\prime}(\cos \beta) .
$$

Close to the extremity of the cone, the first term in the sum is dominant leading to

$$
J_{D} \approx D_{v} a_{1} \sin \beta P_{\ell_{n}}^{\prime}(\cos \beta) r^{\ell_{1}-1} .
$$

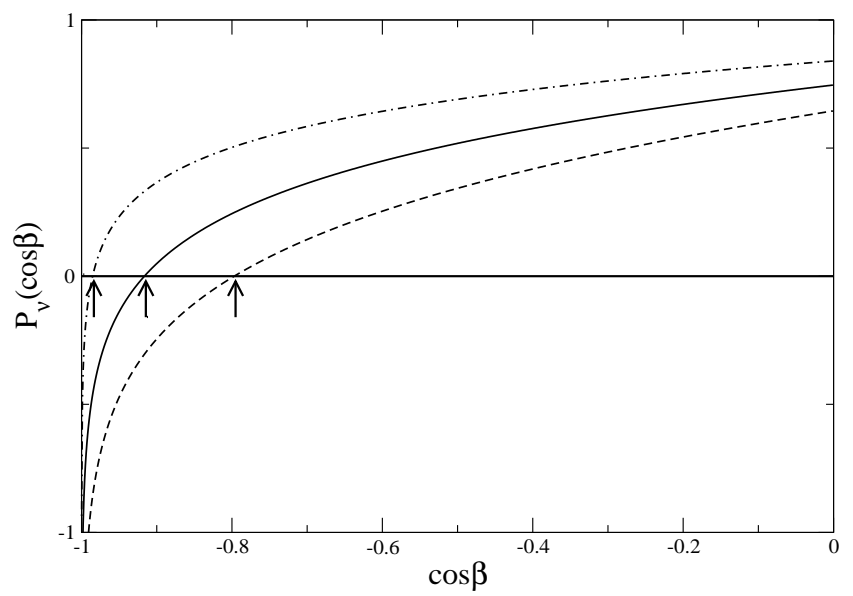

FIG. 3: Plot of the Legendre function $P_{\nu}(\cos \beta)$ versus $\beta$ for $\nu=0.2, \nu=0.3$, and $\nu=0.4$ (from left to right). The arrows indicate the values of $\cos \beta$ corresponding to $P_{\nu}(\cos \beta)=0$. These values correspond to $\beta \approx 170^{\circ}, \beta \approx 156^{\circ}$, and $\beta \approx 143^{\circ}$ (from left to right). The corresponding values of $\nu$ such that $P_{\nu}(\cos \beta)=0$ are $\nu \approx 0.4, \nu \approx 0.3$, and $\nu \approx 0.2$, respectively.

The exponent $\left(\ell_{1}-1\right)$ characterizing the singularity of the behavior at the sharp end is given by the smallest zero of the equation $P_{\ell_{1}}(\cos \beta)=0$. In general, there is no analytical solution to this equation, because $\ell_{1}$ is expected to be non-integer. For $\beta>\pi / 2$, that is, $\cos \beta<0$, the smallest solution of this equation is $\ell_{1} \in[0,1]$. In Fig. 3 we plot $P_{\nu}(\cos \beta)$ versus $\cos \beta$ for various $\nu$. For example, $P_{\nu=0.3}(\cos \beta)$ has a zero at $\cos \beta \simeq-0.91$, which corresponds to $\beta \approx 156^{\circ}$. Hence the solution of $P_{\ell_{1}}(\cos \beta)=0$ for $\beta=156^{\circ}$ is $\ell_{1}=0.3$. Figure 3 also shows that the value of $\cos \beta$ verifying $P_{\nu}(\cos \beta)=0$ goes to -1 as $\nu$ decreases. Hence for $\beta \rightarrow 180^{\circ}$, the value for $\ell_{1}$, which is a solution of the equation $P_{\ell_{1}}(\cos \beta)=0$, goes to zero: $\ell_{1} \rightarrow 0$ as $\beta \rightarrow 180^{\circ}$. This result can be shown more rigorously. In the limit $\nu \rightarrow 0, x \rightarrow-1, P_{\nu}(x)$ may be approximated as $\left.P_{\nu}(x) \simeq 1+\nu \log [(1+x) / 2]\right]^{\underline{5}}$ Therefore, using $\cos \beta \simeq-1+(\pi-\beta)^{2} / 2$ as $\beta \rightarrow \pi$, we deduce that $\ell_{1}$ may be approximated by $\ell_{1} \simeq\left[2 \log \left(\frac{2}{\pi-\beta}\right)\right]^{-1} \stackrel{1}{\underline{1}}$ The important conclusion of these estimates is that for a very sharp cone, $\beta \rightarrow \pi$, the exponent of the singularity at the sharp end is $-1+\ell_{1} \simeq-1$ and thus the flux divergence at the tip scales like $r^{-1}$.

\section{DISCUSSION}

Edge effects lead to a divergent flux of the water vapor at the edges and corners of potatoes. This singular behavior induces a strong drying of the potato near its wedges. This increased dehydration is responsible for the crunchy taste of the potatoes at their wedges.

A few cooking remarks are in order. We have shown that the water flux divergence, and thus dehydration, is stronger as the angle of the corner or cone becomes smaller. The singularity of the water flux is stronger for a cone shape than for a edge: the water flux scales at most as $J_{D} \sim r^{-1 / 2}$ for the two-dimensional wedge (with $r$ the distance to the tip), and $J_{D} \sim r^{-1}$ for a sharp cone. Hence the drying of the potatoes is predicted to be much stronger at the sharp extremities of the wedge than at its edges as is observed (see Fig. 11).

Deep fried wedges exhibit a similar behavior with stronger dehydration at the edges. This similarity may originate in the water vapor bubbles created at the surface of the wedge in hot oil. The above description may apply within the bubbles, although we expect that nonstationary effects of the diffusion process cannot be neglected in such a situation.

Our simple calculations provide an interesting application of the diffusion equation. It has been successful in gaining the interest of the students in a course on the physics of continuum media. Before presenting the calculation in a lecture, students may be asked to perform their own experiment at home and brainstorm on their observations and conclusions. The results may then be debated during the next lecture.

\section{Acknowledgments}

I thank Armand Ajdari for his comments on the manuscript. 
1975), 2nd ed.

2 R. D. Deegan, O. Bakajin, T. F. Dupont, G. Huber, S. R. Nagel, and T. A. Witten, Capillary flow as the cause of ring stains from dried liquid drops, Nature 389, 826-829 (1997).

${ }^{3}$ M. Abramowitz and I. Stegun, Handbook of Mathematical functions (Dover, New York,1972).
${ }^{4}$ Note that the definition of $\beta$ has changed from that in the $2 \mathrm{D}$ case.

5 This relation can be deduced by integrating over $\nu$ the equality $\left.\frac{\partial P_{\nu}(\cos \theta)}{\partial \nu}\right|_{\nu=0}=2 \log [\cos (\theta / 2)]$ (see Eq. (8.6.20) of Ref. 3), and using $P_{0}(x)=1$. 